\title{
VARIATION IN TOTAL POLYPHENOL CONTENT, ANTIOXIDANT POTENTIAL, THEAFLAVIN AND THEARUBIGINS CONTENT OF BOPF GRADE SRI LANKAN BLACK TEAS (Camellia sinensis L.) OF DIFFERENT CLIMATIC ELEVATIONS AND BOPF GRADE COMMERCIALLY AVAILABLE BLACK TEAS IN SRI LANKA
}

\author{
${ }^{1 *}$ R.S. Ratnayake and ${ }^{2}$ K.K.D.S. Ranaweera \\ ${ }^{I}$ Department of Food Science \& Technology, Faculty of Applied Sciences University of Sri Jayewardenepura, \\ Gangodawila, Nugegoda, Sri Lanka \\ ${ }^{2}$ Department of Food Science \& Technology, Faculty of Applied Sciences University of Sri Jayewardenepura, \\ Gangodawila, Nugegoda, Sri Lanka
}

\begin{abstract}
Black tea is the most popular tea among three major types of tea namely green, oolong and black tea. Unblended Sri Lankan black teas are classified as high grown, medium grown and low grown based on their geographical origin. However some studies have been reported regarding the chemical parameters of black teas from different agro climatic elevations of Sri Lanka, such details like purity, cultivar and the particle size of teas have not been specified. And also there is no systematic screening has been reported for black tea commercialized in Sri Lankan market. So the main objective of this study was to analyze the total polyphenol content, antioxidant activity \& total thearubigins \& theaflavins content in unblended Sri Lankan black teas collected from factories representing different tea growing regions. And also to analyze the same biochemical parameters for the black teas that are commercialized in Sri Lankan market. During the month of April 2012, BOPF grade fermented black tea samples produced by TRI 2025 were collected from twelve tea plantations of different agro climatic elevations. All the teas were processed according to the orthodox-rotor vane method. Ten commercialized samples of different brands were also analyzed. Total phenolic content (ISO 14502-1 method), 2,2-diphenyl-1-picrylhydrazyl (DPPH) radical-scavenging activity, TF (flavognost method) and TR content were determined for each sample. Mid grown tea showed highest mean values in TPP content $(15.9454 \pm 2.5778 \%$ GAE), TF content $(22.2200 \pm 3.9575$ $\mu \mathrm{mol} / \mathrm{g})$ and TR content $(14.6031 \pm 1.8970 \%)$. The antioxidant activity was well correlated with the total polyphenol content $(\mathrm{P}<0.05)$ and thearubigins contents $(\mathrm{P}<0.05)$. Lowest mean EC50 value $(49.6791 \pm 5.9475 \mu \mathrm{g} / \mathrm{ml})$ was obtained by mid grown tea samples, indicating highest antioxidant activity among others. Rilagala plantation showed the maximum values in TPP content, antioxidant activity, TF and TR contents among others.
\end{abstract}

Keywords: Black tea, antioxidant activity, total polyphenolic content, theaflavin, thearubigin

\section{INTRODUCTION}

Production of free radicals and reactive oxygen species (ROS) in cells and body tissues have been linked to aging process as well as leading to several human diseases such as diabetes, cirrhosis, cancer cardiovascular diseases and inflammatory disorders (Hertog et al., 1993; Maxwell, 1995). In living organisms, various ROS can form in different ways. Normal aerobic respiration stimulates 
polymorphonuclear leukocytes and macrophages, and peroxisomes appear to be the main endogenous sources of most of the oxidants produced by cells. Exogenous sources of ROS include tobacco smoke, certain pollutants, organic solvents and pesticides (Halliwell, 1994). The antioxidants that scavenge free radicals have major role in the improvement of above diseased conditions.

Antioxidants protect cells against the damaging effects of reactive oxygen species, such as singlet oxygen, super oxide, peroxy radicals, hydroxyl radicals and peroxynitrite which results in oxidative stress leading to cellular damage (Wilson, 1998).

Free radicals generated in the body can be removed by body's own natural antioxidants defenses such as glutathiones and catalases. However, endogenous antioxidant defenses are not completely efficient. Therefore, dietery antioxidants are required to lessen the overall effect of antioxidant stress due to excessive free radicals occurring in our system (Madsen \& Bertelsen, 1995). Synthetic antioxidants such as Butyl hydroxyl anisole (BHA) or butyl hydroxyl toluene (BHT) are used in food industry to decelerate these processes. But, due to their unstable and highly volatile nature, they have been suspected to cause or promote negative health effects (Nabavi $e t$ al., 2008). Therefore there is a trend to substitute them with naturally occurring antioxidants.

Plants are a potential source of natural antioxidants. Natural antioxidants can be phenolic compounds (Flavanoids, phenolic acids and tannins), nitrogen containing compounds (Alkaloids, chlorophyll, derivative amino acids, peptides and amino acids), carotenoids, tocopherols or ascorbic acid and its derivatives and these phytochemical antioxidants are secondary metabolites of plants (Velioglu et al., 1998).

In searching for novel natural antioxidants, some plants have been extensively studied in the past few years for their antioxidant and free radical components (Pourmorad et al., 2006). In this respect presence of flavonoids and other polyphenolic compounds have received the greatest attention (Sundarajan et al., 2006).

Camellia sinensis is commonly known as Tea is one of the most widely consumed beverages in the world and well known to be rich in polyphenolic compound, which plays important roles as antioxidants (Worthy, 1991). The growing interest in the potential health benefits of tea have prompted numerous investigations on the chemical constituents of tea and their biological properties such as anticarcinogenic and antioxidant, antibacterial, anti-inflammatory activities (Gupta et al., 2002). Although a number of mechanisms have been proposed for the beneficial effects of tea, the radical-scavenging and antioxidant properties of tea polyphenols are frequently cited as important contributors to these beneficial effects. Many reports have been studied extensively on the beneficial effects of tea on antioxidant activity and total phenolic content (Gadow et al., 1997).

Many consumers use 'Broken Orange Pekoe Fannings' (BOPF) grade of black tea (a grade containing medium size leaf particles) due to its easy availability and pleasant flavour and taste. However, there is not a great deal of information on the phenolic content, antioxidant activity, theaflavin \& thearubigins content of Sri Lankan BOPF grade black tea for a specific cultivar respect to agro climatic elevation. To date, no previous systematic studies have been reported on the phenolic compounds and antioxidant capacity of commercialized black tea from Sri Lankan market. This is worth examining since it is well recognized that pharmaco-theraputic potential of black tea infusion differs with many factors including country of origin, particle size (or grade of tea), brewing time and agro climatic elevation of sample (Modder and Amarakoon, 2002; Wijeratne, 2008).

The aim of this research was to analyze the total polyphenol content, antioxidant activity \& total thearubigins \& theaflavins content in Sri Lankan black tea originated from three major agro climatic elevations \& black teas commercialized in Sri Lankan market.

Fermented black tea samples of BOPF grade tea produced by TRI 2025 cultivar in same season from four different factories in each elevation representing low-grown (below $600 \mathrm{~m}$, average mean sea level; amsl), midgrown (between 600 and $1200 \mathrm{~m}$, amsl) and high-grown (above $1200 \mathrm{~m}$, amsl) was collected $\&$ analyzed for total polyphenol content, antioxidant activity \& total thearubigins \& theaflavins content. 
And also, ten different black tea samples from different brands commercialized in Sri Lankan market was analyzed for same biochemical parameters.

\section{Main Objective}

To assess the Total polyphenol content, antioxidant activity, total theaflavins \& thearubigin content of Sri Lankan black teas from different agro-climatic elevations \& commercially available black teas from Sri Lankan market.

\section{Specific Objectives}

- To assess the variation in total polyphenol content, antioxidant activity, total theaflavins \& thearubigin content of unblended Sri Lankan black teas \& commercially available black teas (blended) of Sri Lanka.

- To identify the agro-climatic elevation that originates black tea with highest Total polyphenol content, antioxidant activity, total theaflavins \& thearubigin content.

- To determine the correlation between total polyphenol content \& antioxidant activity in above black teas.

- To assess the correlation between Theaflavins/Thearubigins \& antioxidant activity in above black teas.

- To assess the variation in above parameters of each selected garden marks belonging to each agro-climatic region.

\section{LITERATURE REVIEW}

Tea (Camellia sinensis (L.) O. Kuntze) is a member of Theaceae family. It is an evergreen, crosspollinated tree or shrub that can grow to a height of $10-15 \mathrm{~m}$ in the wild, but is usually clipped to a height of 0.6-1.5 $\mathrm{m}$ in cultivation. The leaves are light green, short stalked, coriaceous, alternate, serrate margin, varying in length from $5-30 \mathrm{~cm}$ and about $4 \mathrm{~cm}$ width. Mature leaves are dark green coloured, smooth and leathery while young leaves are pubescent. Flowers are white fragrant, $2.5-4 \mathrm{~cm}$ in diameter, found in solitary or in clusters of two or four. Flowers bear numerous stamens with yellow anther and produce brownish red capsules. Fruit is a flattened, smooth, rounded, capsule with one to five chambers, mostly two or three (Mahmood et al., 2010). Seed is composed of husk, seed capsule, cotyledon and embryo. The seed is rich in fat, starch, sugar with a small amount of saponin. The plant has a strong tap root.

Tea is propagated either from seeds or by cuttings. The tree or shrub is heavily branched with leaves preferentially picked as young leaves and leaf buds to produce tea. Older leaves are considered to be inferior in quality (Sharma et al., 2007).

The two main types of processed tea are black tea and green tea. The difference between these two main types of tea is that black tea manufacture gives full rein to the oxidation of polyphenols by the polyphenoloxidase present in the flush, whilst in green tea manufacture the first operation is the heat destruction of polyphenoloxidase by steaming (Japan) or pan firing (china). It follows therefore, that the primary difference between black tea and green tea is that the former contains a mixture of the native unoxidized polyphenols, together with theaflavin and other oxidized polyphenols only. (Wicramasinghe, 1978)

Sri Lanka produces tea throughout the year and the growing areas are mainly concentrated in the central highlands and southern inland areas of the island. They are broadly grouped under these headings according to their elevations, with high growns ranging from $1200 \mathrm{~m}$ upwards, medium growns covering between $600 \mathrm{~m}$ to $1200 \mathrm{~m}$. and low growns from sea level up to $600 \mathrm{~m}$ (SLTB, 2011)

Four main objectives of tea grading are separation of made tea particles into various shapes and sizes in conformity with trade requirements, size reduction of oversized particles so as to form more acceptable grades, cleaning of grades so as to present them in the most attractive and acceptable manner and preparation for marketing and transport of the produce (Samaraweera and Ziyad, 2008) 
Table 1: Major grades of tea produced in Sri Lanka

\begin{tabular}{|c|c|c|}
\hline Main grade & Sub grade & Description \\
\hline \multirow{2}{*}{$\begin{array}{l}\text { Very special (tips } \\
\text { only) }\end{array}$} & Silver tips & Siler color dried buds \\
\hline & Golden tips & Golden color dried buds \\
\hline Tippy & FBOPF Sp & Similar in size to BOP 1 with a fair presence of tips \\
\hline \multirow[t]{4}{*}{ Flowery } & FBOPF & Similar in size to BOP, BOPF but must contain tips \\
\hline & FBOPF 1 & Larger than BOP, smaller than a FBOP with a show of tips \\
\hline & FBOP & $\begin{array}{l}\text { Smaller/shorter than BOP } 1 \text { with presence of tips, but larger } \\
\text { than FBOPF1 }\end{array}$ \\
\hline & FBOP 1 & Long, twisted, wiry leaf, fairly tippy. Longer than BOP1 \\
\hline \multirow[t]{2}{*}{ Pekoe } & Pekoe 1 & Same as pekoe but smaller in size than pekoe \\
\hline & Pekoe & Shotty, curly or semi-curly leaf of large size \\
\hline \multirow[t]{4}{*}{ Leafy } & BOP 1 & Wiry and twisted, but shorter than OP 1 \\
\hline & OP 1 & Long, wiry, well or partly twisted \\
\hline & OP & Less wiry than OP1, but much more twisted than OPA \\
\hline & OPA & Long bold leaf with fair twist \\
\hline
\end{tabular}

Source: Tea Research Institute, 2008

\section{Chemicals in Tea}

Tea is composed of unique constituents among other plants. Caffeine is found only in a few other plants other than tea. Theanine, which is unique to tea, is a kind of amino acid constituting more than half the total amount of amino acids in tea. Major catechins in tea are also unique to tea. Vitamin $\mathrm{C}$ was found to be contained in tea after it was discovered in lemons. In 1827 caffeine was discovered in tea. At that time it was given the name theine, but when it was proven that the structure and properties of this substance were exactly the same as caffeine that was identified in coffee in 1820, the name theine was dropped (Yukihiko Hara et al., 2001).

In 1924, vitamin C was discovered in green tea by two Japanese scientists, M. Miura and M. Tsujimura. The astringency of tea, too, was investigated extensively by Tsujimura. In the years 1927 to 1935 , Tsujimura isolated epicatechin, epicatechin gallate, and epigallocatechin. She purified them and determined their structural formulas. In 1950, with the new technique of column chromatography, the British scientist A. B. Bradfield succeeded in isolating epigallocatechin gallate and determined its structure by x-ray diffraction method. Tsujimura later identified her compound as being the same. Thus, the main four catechins in tea, which make up the major group of compounds in the soluble solids of tea, were identified in the early 1950s and Tsujimura, along with Bradfield, gained worldwide renowned for their pioneering work (Yukihiko Hara et al., 2001).

Later, around 1963-1965, Y. Takino et al., confirmed the benzotropolone structure of theaflavins. The chemistry of tea polyphenols in that of broader plant polyphenols was well reviewed by E. Haslam. Aroma components in tea were first researched more than 150 years ago by Mulder, who discovered essential oil in fresh tea leaves. In 1930s, Takei and. Yamamoto et al, were among the earliest scientists to contribute to the knowledge of tea aroma. Methods at that time were rather crude and tons of tea, not to mention time and patience, were necessary to isolate sufficient material for separation and identification of individual components. The work of these professors (Takei, who focused mainly on green tea, and Yamamoto, who focused on Taiwan black tea) became a vital basis for future research in the field. They identified more than 30 compounds from green and black teas. Today more than 600 aroma compounds have been identified. Theanine, $\gamma$ ethylamide of glutamic acid, was discovered in 1950 by Sakato. Theanine constitutes the "umami' or sweet taste in tea, particularly that of Gyokuro (the best quality green tea in Japan), and constitutes $2 \%$ of tea. The antagonistic action of theanine against the 
stimulating action of caffeine in the nervous system and its vitalizing action on brain neurons are areas of interest that could be studied further (Yukihiko Hara et al., 2001).

The composition of $C$. sinensis plant varies with climate, season, agricultural practices, varieties of plant, age of leaf, types of leaf and processing methods (Balentine et al., 1998; Yao et al., 2009).

The soluble part of tea consists of polyphenols (mainly catechins) to a large degree, amino acids (theanine), caffeine, minerals and vitamins. The catechin content is up to $30 \%$ of the dry weight, whereas, the content of caffeine is up to $5 \%$ of the dry weight (Balentine, 1997; Graham, 1992).

According to Mukhtar et al., (2000) polyphenols in tea mainly include the following six groups of compounds: flavalonls, hydroxyl-4-flavanols, anthocyanins, flavones, flavonols and phenolic acids. Important and characteristic tea polyphenols are the flavanols of which catechins (flavan-3-ols) are predominant which contribute to the bitterness, astringency and sweet after taste of tea beverages (Hara et al., 1995b). The major ones are: epicatechin (EC), epicatechin gallate (ECG), epigallocatechin (EGC), epigallocatechin gallate (EGCG), catechin (C) and gallocatechin (GC) (Hara et al., 1995a; Liang et al., 2003). Tea also contains favonols, mainly quercetin, kaempferol, myrecetin and their glycosides. In black tea, the enzyme-catalysed oxidation and polymerization of polyphenols during processing, commonly referred to as fermentation, leads to the formation of catechins and gallic acid complexes such as theaflavins and thearubigins, which are responsible for the characteristic dark brown colour in black tea (Balentine, 1997; Lee et al., 2008). In addition, methylxanthines are present with 2 to $4 \%$ as caffeine and as a small amount of theophylline and of theobromine (Hara et al., 1995a). Tea also contains many amino acids, but theanine, specific to the tea plant, is the most abundant, accounting for $50 \%$ of the total amino acids. Balentine (1997) reported that the amino acid degradation is involved in the biogenesis of the tea aroma. Chlorophyll, carotenoids, lipids and volatile compounds present in tea shoots play an important role in the development of the aroma (Hara et al., 1995c).
Volatile fractions of tea leaves have been studied by Hara et al., (1995d) and Shimoda et al., (1995) in detail and more than 600 different molecules have been isolated. These include terpenoids and degradation products of amino acids, carotenoids and linoleic acid (Hara et al., 1995a). Mineral constitutes about 4 to $9 \%$ of the inorganic matter of tea.

According to Hara et al.,(1995c) tea also provides useful amounts of minerals, manganese, potassium and fluoride ions to the diet, and also contains aluminum, iodine, selenium and nickel. This complex composition of tea leaves helps to understand the constituents of tea in particular those that may promote health.

\section{Antioxidant Properties of Tea Polyphenols}

Polyphenols are found in most plants, a diverse group of phenolic compounds (flavanols, flavonols, anthocyanins, phenolic acids, etc.) possess an ideal structural chemistry for free radical scavenging activity (Chanda \& Dave, 2009). Antioxidative properties of polyphenols result from their high reactivity as hydrogen or electron donors from the ability of the polyphenol derived radical to stabilize and delocalize the unpaired electron (chain-breaking function) and from their potential to chelate metal ions (Shahidi \& Wanasundara, 1992; Rice-Evans et al., 1996). However, C.sinensis plant is unique in having huge amount of polyphenols up to $40 \%$ of the solids extracted in to the tea brew). Balentine (2001) reported that tea is one of the richest sources of flavanoids.

\section{Determination of Total Polyphenolic Content}

The amount of total phenol content can be determined by Folin-Ciocalteu reagent method or by Folin-Denis reagent method (Shahidi \& Wanasundara, 1992). Generally this is a basic oxidation-reduction reaction. Mo (40) in Folin's reagent is reduced to $\mathrm{Mo}(40)$ with an electron donated by an antioxidant (Harbone \& Williams,2000). Although the reducing capacity of a substance is not directly related to its radical scavenging capability, it is a very important parameter of antioxidants.

Phenolic compounds react with Folin's reagent only under alkaline conditions and intensity of blue colour produced reflects the amount of phenolic compounds, which can be measured at $765 \mathrm{~nm}$ using 
spectrophotometer (Conforti et al., 2006). However, the exact chemical nature of the Folin's reagent is not known and also the reagent is nonspecific to phenolic compounds as it can be reduced by many non phenolic compounds (vitamin C, $\mathrm{Cu}$ (I), etc.). Despite the undefined chemical nature of the Folin's reagent, this assay is simple, convenient and reproducible. As a result, a large number of data has been accumulated, and it has become a routine assay in studying phenolic antioxidants.

Previously, Povichit et al., (2010) investigated the total phenolic content of ethanolic extracts of fifteen medicinal plants by using Folin-Ciocalteu reagent and found that the leaves of $C$. sinensis plant contains highest phenolic content among the other studied plants. It is generally believed that plants which have more phenolic content show good antioxidant activity that is there is a direct correlation between total phenol content and antioxidant activity (Biglari et al., 2008). Pawin and Sangsrichan (2009) found a positive and strong correlation of total phenolic content and antioxidant activity using ABTS and DPPH assays for Pickled Assam tea leaves ( $C$. sinensis var. assamica), Chineese green tea, Oolong and black tea. It can be stated that phenolic content of the plant may be a good indicator of its antioxidant capacity.

\section{Evaluation of Antioxidant Activity}

A great number of in vitro methods have been developed to evaluate the potency of natural antioxidants either as pure compounds or as plant extracts (Robards et al., 1999; Moure et al., 2001). Antioxidant capacity assays may be broadly classified as hydrogen atom transfer and electron transfer based assays.

Oxygen radical antioxidant capacity (ORAC), total radical trapping antioxidant potential (TRAP) and $\beta$ carotene bleaching test (BCBT) are some of the Hydrogen atom transfer reactions and trolox equivalent antioxidant capacity (TEAC), ferric reducing antioxidant power (FRAP), 1,1-diphenyl-2picrylhydrazyl radical scavenging assay (DPPH), Superoxide anion radical scavenging assay, Hydroxyl radical scavenging assay, Nitric oxide radical scavenging assay are based on electron transfer reactions (Chanda \& Dave 2009).
Different antioxidant evaluating methods do not give the same absolute value but give similar relative rankings to the compound (Modder \& Amarakoon, 2002c). Koleva et al., 2002 carried out a comparative study on three testing methods, DPPH, Static headspace gas chromatography and BCBT to screen out plant extracts for their antioxidant activity and found that the complex composition of the extracts and partition phenomena affected their activity in each assay. According to Salazar et al., (2008) it is essential to use more than one method to evaluate antioxidant capacity of plant materials because of the complex nature of phytochemicals.

A study by Molyneux (2004) reported that the molecule of 1,1-diphenyl-2-picrylhydrazyl ( $\alpha, \alpha$ diphenyl- $\beta$-picrylhydrazyl) is characterized as a stable free radical as they do not dimerise. The delocalization of the spare electron over the molecule also gives rise to the deep violet colour. When a solution of DPPH in ethanol is mixed with that of a substance that can donate a hydrogen atom, gives rise to the formation of reduced form of DPPH. This transformation results in colour change from violet to yellow and usually measured spectropheotometrically at $517 \mathrm{~nm}$ (Chanda \& Dave, 2009).

\section{Theaflavins and Thearubigins Determination in Black Tea}

Approximately $76-78 \%$ of the tea produced and consumed worldwide is black tea (Cabrera et al., 2003). During the black tea manufacture, a natural enzyme catalyzes oxidation and condensation of green leaf tea catechins leading to the formation of theaflavins (TF) and thearubigins (TR) (Robertson, 1992).

The plain black teas are evaluated on the basis of their briskness, brightness, strength, body and total colour of liquors (Roberts \& Smith, 1963). Black tea liquor brightness and colour are critical quality attributes used in tea trade to rank and price black teas (Biswas, Sarkar, \& Biswas, 1973; McDowell, Feakes, \& Gay, 1991). These attributes of black tea quality are mainly dictated by the levels of theaflavins and thearubigins (Robertson, 1992). Theaflavins are responsible for the astringency, brightness, colour and briskness of the black tea. Thearubigins contribute to the mouth feel (thickness) 
and colour of the tea (Biswas, Biswas, \& Sarkar, 1973).

Black tea quality is mainly influenced by total theaflavins (Wright, Mphangwe, Nyirenda, \& Apostolides, 2002) or derived theaflavin digallate equivalents (Owuor \& Obanda, 1997).

The formation of a single theaflavin requires a dihydroxy and trihydroxy flavan-3-ols. The ratio of dihydroxyflavan-3-ol to trihydroxy-3-ol in green leaf may thus have a major influence on the amount of theaflavins in black tea. The correct balance and amount of dihydroxyflavan-3-ol and trihydroxyflavan-3-ol are therefore necessary to ensure maximum formation of the theaflavins (Wright et al., 2002). The amount of the individual theaflavins are formed largely influenced by the amounts of the precursor catechins in green leaf, their redox potential and/or affinity for polyphenol oxidase and activity (Owuor \& Obanda, 2007).

Fermentation is a critical stage in the manufacture of black tea during which oxidative condensation of catechins to TF and TR occurs. Catechins, together with their oxidation products are responsible for most of the sensory characteristics associated with black tea liquors (Biswas et al., 1973). Temperature and time are important factors in determining the extent of fermentation. Processing conditions which favour less degradation of simple theaflavins and the retention of higher epicatechin gallate (ECG) and epigallocatechin gallate (EGCg) levels produce more brisk tea liquors (Obanda, Owuor, \& Mang'oka, 2001). Theaflavins and unoxidized catechins are thought to have considerable human health benefits (Apostolides \& Weisberger, 1995). Therefore, tea clones or processing conditions with a high potential of achieving this, can enhance the health benefit potential of black tea and impact positively on liquor astringency.

The formation and contribution of TR to quality characteristics of black tea have also been reported but their structures remain speculative. In 1961, Roberts and Smith demonstrated TR could separate into two large groups, i.e. TRSI and TRSII, due to differences in chemical polarities. Roberts (1962) reported that TR was acidic brown pigments formed by the oxidative degradation of TF.
Traditional methods for the preparation and determination of polyphenols in fresh tea shoots or manufactured teas have been described by several researchers (Roberts, 1962; Roberts \& Myers, 1958; Roberts \& Smith, 1961). The most common methods are paper chromatography (Roberts, Cartwright, \& Wood, 1956), column chromatography (Oshima \& Nakabayashi, 1953; Whitehead \& Temple, 1992) and colorimetric measurement (Muralidharan, 1997; Oshima \& Nakabayashi, 1953; Roberts \& Smith, 1961). All those methods are based on either the oxidation or reduction properties of tea polyphenols.

More recently developed analytical techniques are used to isolate, identify and determine individual polyphenolic compounds by HPLC (Harbowy \& Balentine, 1997; Temple \& Clifford, 1997; Yao \& Nursten, 1997, 1998). However, colorimetric or spectrophotometric methods are still the most widely used, due to their simplicity for the determination of total phenolic compounds, TF and TR in tea (Harbowy \& Balentine, 1997; Lakenbrink et al., 2000), particularly for tea industries.

The method developed for determining theaflavins and thearubigins depends on the fact that the theaflavins are almost quantitatively extracted from tea liquor by one extraction with either ethyl acetate or isobutyl methyl ketone. These solvents do not extract thearubigins of the S II type, but there is a partial extraction of the free-acid forms of the S I type thearubigins. Potassium and calcium salts are not extracted. The thearubigins extracted by ethyl acetate or isobutyl methyl ketone are soluble in aqueous sodium hydrogen carbonate, whereas the theaflavins are insoluble. Complete separation of theaflavins and thearubigins is therefore effected by shaking the ethyl acetate or isobutyl methyl ketone extract with aqueous sodium hydrogen carbonate.

Theaflavin and its gallate have well defined absorption maxima at 380 and $460 \mathrm{m \mu}$. (Roberts, and Myers, 1959). Either of these wavelengths is suitable for direct spectrophotometry in the extract washed with sodium hydrogen carbonate, as no other substances are present that absorb at these wavelengths. The fall in optical density, which results from the washing with sodium hydrogen carbonate, affords a method of determining the extractable thearubigins. 
Direct spectrophotometric determination of residual thearubigins in the aqueous layer after extraction with ethyl acetate or isobutyl methyl ketone is not possible, as a high proportion of the thearubigin molecules are present as anions, which are more deeply coloured than the free acids. Addition of excess aqueous oxalic acid reduces the colour intensity to that of the free acids, and spectrophotometry is possible after this acidification.
Fresh tea samples of TRI 2025 were collected separately from each selected plantation during April 2012, and undergone to an orthodox rotor vane process. About $200 \mathrm{~g}$ of BOPF grade processed black tea sample from each factory on completion of processing were collected. The plantations were selected to represent the key tea producing regions in Sri Lanka. (See Table 1)

\section{METHODOLOGY}

\section{Samples}

Table 2: Names, Locations and sampling periods for the Sri Lankan Teas

\begin{tabular}{|c|c|c|}
\hline $\begin{array}{l}\text { Tea } \\
\text { growing } \\
\text { region }\end{array}$ & Plantation & Description of the location \\
\hline \multirow{4}{*}{$\begin{array}{l}\text { High } \\
\text { grown } \\
(>1200 \mathrm{~m})\end{array}$} & Holyrood & $\begin{array}{l}\text { Located in western slopes of the central hills of Dimbulla planting } \\
\text { district, about } 1340 \mathrm{~m} \text { above sea level }\end{array}$ \\
\hline & Somerset & $\begin{array}{l}\text { Located in western slopes of the central hills, situated in the lower } \\
\text { boundary of the misty village known as Nanuoya, about } 1200 \mathrm{~m} \\
\text { above sea level }\end{array}$ \\
\hline & Greatwestern & $\begin{array}{l}\text { Situated in the foothills of the majestic Great Western range, about } \\
1200-1800 \mathrm{~m} \text { above sea level }\end{array}$ \\
\hline & Mattakelle & $\begin{array}{l}\text { Located in western slopes of the central hills, about } 1400 \mathrm{~m} \text { above } \\
\text { sea level }\end{array}$ \\
\hline \multirow{4}{*}{$\begin{array}{l}\text { Mid } \\
\text { grown } \\
(1200- \\
600 \mathrm{~m})\end{array}$} & Craighead & $\begin{array}{l}\text { Located in the middle slopes about } 900 \mathrm{~m}-1100 \text { above sea levels. } \\
\text { There are no seasonal variations in harvesting patterns for mid } \\
\text { grown teas }\end{array}$ \\
\hline & Imboolpitiya & Located at an elevation about $800 \mathrm{~m}$ above sea levels. \\
\hline & Dombagastalawa & Located at an elevation about above m600-900 m sea levels. \\
\hline & Rilagala & Located at an elevation about $1000 \mathrm{~m}$ above sea levels. \\
\hline \multirow{4}{*}{$\begin{array}{l}\text { Low } \\
\text { grown } \\
(<600 \mathrm{~m})\end{array}$} & Houpe & $\begin{array}{l}\text { Located at an elevation of about } 550 \mathrm{~m} \text { above sea level. There are } \\
\text { no seasonal variations in harvesting patterns for low grown teas }\end{array}$ \\
\hline & Rilhena & Located at an elevation of about $500 \mathrm{~m}$ above sea level. \\
\hline & Samawatte & Located at an elevation of about $450 \mathrm{~m}-500 \mathrm{~m}$ above sea level. \\
\hline & Wellandara & Located at an elevation of about $500 \mathrm{~m}$ above sea level. \\
\hline
\end{tabular}

Ten commercial bagged tea samples of different brands (A-J) were purchased from a Sri Lankan supermarket. When purchasing bagged tea products, they were selected with similar manufactured date.
For each commercial tea sample studied, three bags were sampled.

\section{Chemicals}

Analytical Reagent Grade Absolute Methanol (Prolabo-UK), Analytical reagent grade sodium 
carbonate anhydrous (Fisher Scientific, UK), Folinciocalteu phenol reagent (Prolabo-UK), Gallic acid (Sigma Aldrich Co. USA), Analytical Reagent Grade Absolute Ethanol (Prolabo-UK), DPPH (2,2Diphenyl-1-picrylhydrazyl), IBMK (isobutyl methyl ketone), Flavognost Reagent (diphenylboric acid 2aminoethyl ester) were purchased from Sigma Aldrich- USA.

\section{Extraction of Polyphenols}

The method described by the International Organization for Standardization (ISO) 14502-1 was used. Briefly, 0.200 ( $0.001 \mathrm{~g}$ of each sample was weighed in an extraction tube, and $5 \mathrm{~mL}$ of $70 \%$ methanol at $70{ }^{\circ} \mathrm{C}$ was added. The extract was mixed on a vortex for $10 \mathrm{~min}$ and heated at $70{ }^{\circ} \mathrm{C}$. After cooling at room temperature, the extract was centrifuged at $3500 \mathrm{rpm}$ for $10 \mathrm{~min}$. The supernatant was decanted into a graduated tube. The extraction step was repeated twice. Both extracts were pooled and the volume adjusted to $10 \mathrm{~mL}$ with cold $70 \%$ methanol. One millilitre of the extract was diluted with water to $100 \mathrm{~mL}$.

\section{Estimation of the Total polyphenol content by Folin-Ciocalteu assay}

The total polyphenol content (TPC) was determined by spectrophotometry, using Gallic acid as standard, according to the method described by the International Organization for Standardization (ISO) 14502-1. Briefly, $1.0 \mathrm{~mL}$ of the diluted sample extract was transferred in duplicate to separate tubes containing $5.0 \mathrm{~mL}$ of a $1 / 10$ dilution of FolinCiocalteu's reagent in water. Then, $4.0 \mathrm{~mL}$ of a sodium carbonate solution $(7.5 \% \mathrm{w} / \mathrm{v})$ was added. The tubes were then allowed to stand at room temperature for $60 \mathrm{~min}$ before absorbance at $765 \mathrm{~nm}$ was measured against water. The TPC was expressed as Gallic acid equivalents (GAE) in $\mathrm{g} / 100 \mathrm{~g}$ material. The concentration of polyphenols in samples was derived from a standard curve of Gallic acid ranging from 10 to $50 \mu \mathrm{g} / \mathrm{mL}$ (Pearson's correlation coefficient: $r^{2}=0.9910$ ).

\section{Determination of the Free Radical Scavenging Activity by DPPH Free-Radical Scavenging Assay}

Scavenging activities of the extracts on the stable free radical DPPH were assayed using the modified Blois' method, in which the bleaching rate of DPPH is monitored at a characteristic wavelength in presence of the sample.

The diluted working solutions of the test extracts were prepared $(1.00,5.00,10.00,20.00,30.00$ $\mu \mathrm{g} / \mathrm{ml}) .0 .04 \% \mathrm{w} / \mathrm{v}$ of DPPH was prepared in absolute ethanol and $2 \mathrm{ml}$ of this solution was mixed with $2 \mathrm{ml}$ of sample solution separately. These solution mixtures were kept in dark for $30 \mathrm{~min}$ and optical density was measured at $517 \mathrm{~nm}$ using UV-mini Spectrophotometer. Ethanol $(2 \mathrm{ml})$ with DPPH solution $(0.04 \%, 2 \mathrm{ml})$ was used as blank. The optical density was recorded and \% inhibition was calculated using the formula given below:

$\mathrm{I} \%=\mathrm{A}_{\text {control }}-\mathrm{A}_{\text {sample }} \times 100 \%$
$\mathrm{~A}_{\text {control }}$
$\mathrm{A}_{\text {control }}=$ Absorbance of the control
$\mathrm{A}_{\text {sample }}=$ Absorbance of the sample / ascorbic acid
solution

Total TF content analysis (Flavognost Method)

Total TF were determined by the Flavognost method (Hilton, 1973). A tea infusion was made with $375 \mathrm{ml}$ of boiling water, added from an overhead boiler into a tared flask, and $9 \mathrm{~g}$ of tea. The flask was shaken for $10 \mathrm{~min}$, the infusion filtered rough cotton wool, and allowed to cool to room temperature, and then $10 \mathrm{ml}$ were pipetted into $10 \mathrm{ml}$ of isobutylmethylketone (4methylpentan-2-one, IBMK). The mixture was shaken for $10 \mathrm{~min}$ and allowed to stand until the layers separated. Two millilitres of the upper layer were pipetted into a test tube, followed by $4 \mathrm{ml}$ ethanol and $2 \mathrm{ml}$ Flavognost reagent (2 $\mathrm{g}$ diphenylboric acid-2-aminoethyl ester dissolved in $100 \mathrm{ml}$ ethanol). The contents were mixed and colour allowed $15 \mathrm{~min}$ to develop. The absorbance (A) at $625 \mathrm{~nm}$ was read against an IBMK/ethanol (1:1 v/v) blank. 



\section{Theaflavin content $(\mu \mathrm{mol} / \mathrm{g})=\mathrm{A}_{625 \mathrm{~mm}} \times 47.9 \times 100 / \mathrm{DM}$}

\section{Determination of Total Thearubigins}

Fifty milliliters of the cool, well-shaken and filtered standard tea infusion from TF analysis were mixed with $50 \mathrm{ml}$ isobutyl methyl ketone (IBMK) and gently shaken to avoid formation of an emulsion. The layers were allowed to separate and 4-ml portion of the IBMK layer was taken and made to $25 \mathrm{ml}$ with methanol in a volumetric flask (Solution A). Two milliliter portions of the aqueous layer were diluted to $10 \mathrm{ml}$ with distilled water and then to $25 \mathrm{ml}$ with methanol (Solution B). Twenty-five milliliters of the remaining initial IBMK layer were taken in a separate flask and mixed with $25 \mathrm{ml}$ of $2.5 \%$ aqueous sodium hydrogen carbonate. The mixture was vigorously shaken before the layers were allowed to separate and aqueous layer discarded. A 4-ml portion of the washed IBMK layer was made to $25 \mathrm{ml}$ with methanol (Solution C). Two milliliters of a saturated oxalic acid aqueous solution and $6 \mathrm{ml}$ of water were added to a 2-ml portion of the aqueous layer left from the first extraction with IBMK, and diluted to $25 \mathrm{ml}$ with methanol (Solution D).

The absorbencies $A_{A}, A_{C}, A_{D}$ of solutions $A, C$ and $D$ at $380 \mathrm{~nm}$ were obtained using a spectrophotometer (UV- mini, Shimadzu) with distilled water as the blank. Each black tea sample was extracted in triplicate for the determination of the Total Thearubigins content.

$$
\begin{aligned}
& \text { \% Thearubigin Content (Total) } \\
& \qquad=\left(\frac{375 \times 0.02 \times 6.25 \times\left[2 A_{D}+A_{A}-A_{C}\right]}{0.733 \times 9 \times D M / 100}\right)
\end{aligned}
$$

\section{DATA ANALYSIS}

All determinations were carried out in three triplicate and data were subjected to analysis of variance. Analysis of variance was performed using the ANOVA procedure. The data were normally distributed at the $95 \%$ confidence level and Anderson-Darling test was used for the determination of normality of the data. Two normality assumptions were made for the residuals at the $95 \%$ confidence level according to the $\mathrm{p}$ value of the test parameters. $P$ values less than 0.05 were considered statistically significant. Significant differences between means were determined by Tukey test. $P$ values less than 0.05 were considered statistically significant. TwoSample-T Test was carried out to analyze the significance of the tested parameters between black tea samples from different agro climatic elevations and commercial black tea samples. All statistical analyses were performed using the MINITAB14 software.

\section{RESULTS \& DISCUSSION}

According to the results, mid grown tea had showed highest mean values in TPP content (15.9454 \pm $2.5778 \%$ GAE), TF content $(22.2200 \pm 3.9575$ $\mu \mathrm{mol} / \mathrm{g})$ and TR content $(14.6031 \pm 1.8970 \%)$. And also the lowest mean $\mathrm{EC}_{50}$ value $(49.6791 \pm 5.9475$ $\mu \mathrm{g} / \mathrm{ml}$ ) was obtained by mid grown tea samples, which indicated the highest antioxidant activity by scavenging DPPH free radicals. For TPP content \& $\mathrm{EC}_{50}$ value, there is no significance difference showed between means of mid grown and low grown teas.

The results of antioxidant activity were much similar to the trend of total polyphenolic content in different black tea samples and showed a positive linear correlation between total polyphenolic content and $\mathrm{EC}_{50}$ values $(\mathrm{P}=0.018)$. There was no correlation was found between Theaflavins content and antioxidant activity. But a positive linear correlation 
between antioxidant activity and TR content $(\mathrm{P}=$ 0.037) was found.

Black tea samples from Rilagala plantation of mid grown region was found to be the finest with respect to TPP content (18.6595 $\pm 0.6977 \%$ GAE), antioxidant activity $\left(\mathrm{EC}_{50}=42.2706 \mu \mathrm{g} / \mathrm{ml}\right)$ and $\mathrm{TR}$ content (16.9016 $\pm 0.1261 \%)$; followed by Imboolpitiya plantation of mid grown region and Samawatte plantation of low grown region.

From two sample T-test results, it is found that there was significant difference between the TPP content $(\mathrm{P}<0.05)$, TF content and TR content $(\mathrm{P}<0.05)$ of black tea samples from different agro climatic elevations and commercial black teas.

\section{CONCLUSION}

The study demonstrates considerable variation among both unblended and commercialized Sri Lankan black tea samples for TPP, antioxidant properties as determined by DPPH, TF and TR contents. It clearly shows that there is considerable potential for using selected combinations of plantations and season to select high antioxidant teas.

The results of antioxidant activity were much similar to the trend of total polyphenolic content in different black tea samples and showed a positive linear. A positive linear correlation between antioxidant activity and TR content was also found. There was also evidence for an interaction effect for TPP with DPPH among plantations. Various reasons may be behind these differences such as climate and soil fertility. In terms of selecting high antioxidant teas, attention needs to be paid to plantation and the growing region.

This study indicates that the bioactive molecules present in black tea produced by $C$. sinensis can be used as a valuable antioxidant source. Further studies to elucidate the role of respective polyphenols in tea responsible for antioxidant activity are very promising.

\section{ACKNOWLEDGEMENT}

This research was supported financially by Ceylon Tea Services PLC, Peliyagoda, Sri Lanka. Authors wish to thank Ms. Harshini
Rajakulendran \& Mr. Indika Palagasingha of Ceylon Tea Services PLC for their excellent assistance in this study.

\section{REFERENCES}

Akio Kobayashi, Hisako Sato, Royoko Arikawa and Tei Yamanishi (1965), Flavour of Black tea, Journal of Agr. Biol. Chem, Vol.29,No.10, pp.902-907.

Alain Chaintreau (2000), Simultaneous distillation and extraction from birth to Maturity review, Journal of Flavour and Fragrance, vol. 16: 136-148

Anthony J. Wright and Michael J. fishwick (1978), Lipid Degradation During manufacture of black tea, Journal of Phytochemistry, Vol.18,pp, 1511-1513.

Biswas A.K., Biswas A.K., and Sarkar A.R, (1973), "Statistical Evaluation of the Biochemical Constituents and their Effects on Colour, Brightness and Strength of Black Teas", Journal of the Science of Food and Agriculture, Volume 24, Issue 12, pp. 1457-1477, December 1973

Chi-Tang Ho, Jen-Kun Lin, Fereidoon Shahidi (1944), Tea and tea products, chemistry and health-promoting properties, Memorial University of Newfoundland, St. John's, Newfoundland, Canada.

Davies K. J. Oxidative stress: the paradox of aerobic life Biochem. Soc. Symp. 61, 31, 1995

De Silva, Rasangika Sri Lankawe te wagawe arambaya, Samantha publishers, $1^{\text {st }} \mathrm{ed}, 2005,27-30$

Frenkel K., Chrzan K. Hydrogen peroxide formation and DNA base modification by tumor promoter-activated polymorphonuclear leukocytes Carcinogenesis 8, 455, 1987

Gupta, S., Saha, B., \& Giri AK (2002). Comparative antimutagenic and anticlastogenic effects of green tea and black tea: a review. Mutat. Res, 512(1), 37-65.

Gadow, V.A. Joubert, E., \& Hansmann, C.F. (1997). Comparison of the antioxidant activity of rooibos with green, oolong, and black tea. Food Chem, 60(1), 73-77.

Ghiselli A., Serafini M., Natella F., Scaccini C. Total antioxidant capacity as a tool to assess redox status: critical view and experimental data. Free Rad. Biol. Med. 29, 1106, 2000

Halliwell B, Gutteridge J. M. C., Cross C. E. Free radicals, antioxidants and human disease: where are we now? J. Lab. Clin. Med. 119, 598, 1992

Halliwel, B. (1994). Free radicals, Antioxidants, and human disease: Curiosity, Cause, or Consequence? Lancet, 344, 721-724.

Harbowy ME, Balentine DA (1997). Tea chemistry. Critical Rev. Food Sci. Nutrition. Volume 16, pg. 415-480

Hertog, M.G. Feeskens, E.J., Hollman, P.C., Katan, M.B., \& Kromhout, D. (1993). Dietery antioxidant flavonoids and risk of coronary heart disease: the Zutphen Elderly Study. Lancet, 342, 1007-1011.

History of tea, http://en.wikipedia.org/wiki/History_of_tea_in_China, viewed $14^{\text {th }}$ December 2012. 
Jose A. B. Baptista, Joaquim F. da P Tavares and Rita C. B. Carvalho (1998), Comparison of catechins and aromas among different green teas using HPLC/SPME-GC, Journal of Food Research International, Vol. 31, No. 10, pp. $729 \pm 736$

Kinsella J. E., Frankel E., German B., Kanner J. Possible mechanism for the protective role of antioxidants in wine and plant foods Food Technol. 47, 85, 1993

Lakenbrink, C., Lapczynski, S., Maiwald, B., Engelhardt, U.H., 2000. Flavonoids and other polyphenols in consumer brews of tea and other caffeinated beverages. Journal of Agricultural and Food Chemistry 48, pg. 2848-2852

Leopold Jirovetz, Gerhard Buchbauer, Martin Benoit Ngassoum, Margit Geissler (2002), Aroma compound analysis of Piper nigrum and Piper guineense essential oils from Cameroon using solid-phase microextraction-gas chromatography, solid-phase microextraction-gas chromatography- mass spectrometry and olfactometry, Journal of Chromatography A, vol. 976 (2002) 265-275.

Liu, Y., Guo, Y., and Zheng, Z. (2003), Investigations on the variation of main chemical substances and the formation of white tea during manufacturing. J. Fujian Tea.

Li-Fei Wang a, Joo-Yeon Lee b, Jin-Oh Chung a, Joo-Hyun Baik a, Sung So a, Seung-Kook Park b(2006), Discrimination of teas with different degrees of fermentation by SPME-GC analysis of the characteristic volatile flavour compounds, Journal of Food Chemistry, vol. 109 (2008) 196-206.

Madsen, H.L., \& bertelsen, G. (1995). Spices as antioxidants. Trends Food Sci. Technol, 6, 271-277

McDowell, I., Feakes, J., Gay, C., 1991, Phenolic composition of black tea liquors as a means of predicting price and country of origin, Journal of the Science of Food and Agriculture, Volume 55, Issue 4, pp. 627-641

Modder, W.W.D., \& Amarakoon, A.M.T. (2002a). The chemicals in tea shoots and their health effects, In Tea \& Health (pp13). The Tea Research Institute in Sri Lanka.

Modder, W.W.D., \& Amarakoon, A.M.T. (2002b). Tea \& cancer, In Tea \& Health (pp103-104). The Tea Research Institute in Sri Lanka.

Modder, W.W.D., \& Amarakoon, A.M.T. (2002c). Antioxidant Activity, In Tea \& Health (pp 61-64). The Tea Research Institute in Sri Lanka.

Muralidharan, D. (1997). Spectrophotometric analysis of catechins and condensed tannins using Ehrlichs reagent. Journal of the Society of Leather Technologists and Chemists, Volume 81, pg. 231-233

Nabavi, S.M., Ebrahimzadeh, M.A., Nabavi, S.F., \& Jafari, M. (2008). Free radical scavenging activity and antioxidant capacity of Eryngium caucasicum Trautv and Froripia subpinnata. Pharmacologyonline, 3, 19-25

N. Togari, A. Kobayashi' \& T. Aishima (1995), Pattern recognition applied to gas chromatographic profiles of volatile components in three tea categories, Journal of Food Research International, Vol. 28, No. 5, pp. 495-502

Ninomiya M., Unten L., Kim M. Chemical and physicochemical properties of green tea polyphenols; in Chemistry and applications of green tea, ed. Yamamoto T., Juneja L. R., Chu D. C., Mujo K., CRC Press, New York pp 23-35, 1997
Obanda, M. and Owuor, P.O., 1992. Effect of wither and plucking on the biochemical and chemical parameters of selected Kenyan tea. Discov.Innov. 6, pp. 190-197.

Obanda, M., Owuor, P. O., \& Taylor, S. J. (1997). Flavanol composition and caffeine content of green leaf as quality potential indicators of Kenyan black teas. Journal of the Science and Food Agriculture, Volume 74, pp. 209-215.

Obanda, M., Owuor, P.O. And Mang'oka, R. (2001), Changes in the chemical and sensory quality parameters of black tea due to variations of fermentation time and temperature, Food Chem, Volume 75, pg. 395-404

Oshima, Y., \& Nakabayashi, T. (1953a). Studies on catechins and pigments in tea leaves. Part 5. Paper chromatography of tea flavones. Journal of Agricultural Chemistry, Japan, Volume 27, pg. 754-756.

Oshima, Y., \& Nakabayashi, T. (1953b). Studies on catechins and pigments in tea leaves. Part 6. Isolation of tea flavones by column chromatography. Journal of Agricultural Chemistry, Japan, Volume 27, pg. 756-759.

Oshima, Y., \& Nakabayashi, T. (1953c). Colorimetric estimation of gallic acid and flavonoid pigments in tea leaves. Journal of Agricultural Chemistry, Japan, Volume 26, pg. 377-381

Ostrowska J., Stankiewicz A., Skrzydlewska E. Antioxidative properties of green tea. Bromatol. Toxicol. Chem. 2, 131, 2001

Pourmorad, F., Hosseeinimehr, S.J. \& Shahabimajd, N (2006). Antioxidant activity, phenol and flavonoid contents of some selected Iranian medicinal plants. Afr. $J$. Biotechnol, 5, 1142-1145

Renu Rawat, Ashu Gulati, G.D. Kiran Babu, Ruchi Acharya, Vijay K. Kaul, Bikram Singh (2006), Characterization of volatile components of Kangra orthodox black tea by gas chromatography-mass spectrometry, Journal of Food Chemistry, vol.105 (2007) 229-235.

R. L. Wickremasinghe, Emily L. Wick and Tei Yamanishi (1972),Gas chromatographic -mass spectroscopic analysis of "Flavory" and "non flavor" Ceylon Black tea aroma concentrates prepared by two different methods, Journal of Chromatography, vol. 79 (1973) 75-80

R.L. Wickremasinghe (1978), Monograph on Tea Production in Sri Lanka, Tea Research institute of Sri Lanka.

Robertson, A. (1992). The chemistry and biochemistry of black tea production - the non-volatiles. In: K.C. Willson (Ed.) \& M.N. Clifford, Tea: Cultivation to consumption, (pg. 574-580). Kluwer Acad. Publ., Dordrecht, The Netherlands.

Roberts, E. A. H., (1956), The leuco-anthocyanins of unprocessed tea-leaf, Journal of the Science of Food and Agriculture, Volume 7, Issue 4, pages 253-257.

Roberts, E. A. H., Cartwright, R. A., \& Oldschool, M. (1957), The phenolic substances of manufactured tea, Journal of the Science and Food Agriculture, Volume 8, pg. $72-80$.

Roberts, E. A. H., and R. F. Smith (1961), Spectrophotometric measurements of theaflavins and thearubigins in black tea liquors in assessments of quality in teas, Analyst, Volume 86, Issue 1019, pg. 94-98 
Robert R. Selvendran, John Reynolds and Terence Galliard (1977), Production of Volatiles By Degradation of Lipids During Manufacture Of Black Tea, Journal of Phytochemistry, Vol.17,pp,233

Roy Teranishi and Robert A. Flath (1981), Flavour Research (Resent advances), Western regional Research center, Agriculture Research Service, California

Ryoyasu Saijo (1967), Volatile Flavour of Black Tea, Journal of Agr. Biol. Chem., Vol.31, No.11, p.1265-1269.

Schlesier K., Harwat M., Bohm V., Bitsch R. Assessment of antioxidant activity by using different in vitro methods Free Radic. Res. 36, 177, 2002

Spiro Michael and William E. Price, Determination of theaflavins in tea solution using the flavognost complexation method, Analyst, 1986,111, 331-33

Sundarajan, R., Haja, N., Venkatesan, K., Mukherjee, K., Saha, B., Bandyopadhyay, A., \& Mukherjee, P. (2006). Cytisus scoparius link - Anatural antioxidant. BMC Complement. Altern. Med, 6, 8-14.

Tei Yamanishi, Alio kobayashi, Hisako Sato, Akiko Ohmura and Hideko Nakamura (1965), Flavory of black tea, Journal of Agr. Biol. Chem, Vol.29,No.11, p.10161020.

Tei Yamanishi, Michiko Nose and Yoichi Nakatani (1970), Studies on the flavour of green tea, Journal of Agr. Biol. Chem, Vol. 34, No.4, p.599-608.

Tei yamanishi, Akio Kobayashi, Atsuko Uchida and Yoko Kawashima (1996), Studies on the flavour of green tea, Journal of Agr. Biol. Chem, Vol 30, No.11, p. 1102-1105.

Temple, C. M., \& Clifford, M. N. 1997. The stability of theaflavins during HPLC analysis of a decaffeinated aqueous tea extract. Journal of Agricultural and Food Chemistry, 74, pp. 536-540.

The Chemistry of Tea, http://nobleharbor.com/tea/teachemistry.html, viewed $13^{\text {th }}$ November 2012
Velioglu, Y.S., Mazza., G, Gao, L., \& Oomah, B.D. (1998). Antioxidant activity and total phenolics in selected fruits, vegetables, and grain products. J. Agric. Food Chem, 46(24), 4113-4117.

Whitehead, D. L., and Temple, C. M., 1992. Rapid method for measuring thearubigins and theaflavins in black tea using C18 absorbent cartridges. Journal of the Science and Food Agriculture, 58, pp. 149-152

Wright, L.P., Mphangwe, N.I.K., Nyirenda, H.E. and Apostolides, Z., 2002. Analysis of the theaflavin composition in black tea (Camellia sinensis) for predicting the quality of tea produced in Central and Southern Africa, Journal of the Science of Food and Agriculture, 82, pp. 517-525.

Wilson, R.L. (1998). Free radicals and tissue damage, mechanistic evidence from radiation studies. In" Biochemical Mechanisms of Liver Injury, (pp.12), Academic Press, New York.

Worthy, W. (1991). Fruit, vegetables, green tea may cut cancer risk. Chem. Eng, 69(37), 27-29

Yang Zhao, Pei Chen, Longze Lin, J.M. Harnly, Liangli (Lucy) Yu, Zhangwan Li (2010), Tentative identification, quantitation, and principal component analysis of green puerh, green, and white teas using UPLC/DAD/MS,Journal of Food Chemistry, vol. 126 (2011) 1269-1277

Yao, L. H., \& Nursten, H. E. (1997). The chemical oxidation of individual (-)-epicatechin gallate and (-)epigallocatechin gallate. Journal of Food and Fermentation and Industry, Volume 23, pg. 21-25.

Yao, L. H., \& Nursten, H. E. (1998). Reversed-phase HPLC with photodiode array detection of the oxidation products from (-)- ECG, (-)-EGCG and black tea liquor. Journal of Food and Fermentation and Industry, Volume 24, pg. 11-16.

Yuerong Liang*, Jianliang Lu, Lingyun Zhang, Shan $\mathrm{Wu}$, Ying Wu (2002), Estimation of black tea quality by analysis of chemical composition and colour difference of tea infusions, Journal of Food Chemistry, vol. 80 (2003) 283-290.

Yukihiko Hara(2001), Green tea, health benefits and applications, Tokyo Food Techno Co., Lt, Tokyo, Japan. 